\title{
Multi-components ensembles of future meteorological and natural snow conditions in the Northern French Alps Supplementary figures
}

Deborah Verfaillie ${ }^{1}$, Matthieu Lafaysse ${ }^{1}$, Michel Déqué ${ }^{2}$, Nicolas Eckert ${ }^{3}$, Yves Lejeune ${ }^{1}$, and Samuel Morin $^{1}$

${ }^{1}$ Météo-France - CNRS, CNRM UMR 3589, Centre d'Études de la Neige, Grenoble, France

${ }^{2}$ Météo-France - CNRS, CNRM UMR 3589, Toulouse, France

${ }^{3}$ Université Grenoble Alpes, Irstea, UR ETGR, Grenoble, France

Correspondence to: Samuel Morin, samuel.morin@meteo.fr 


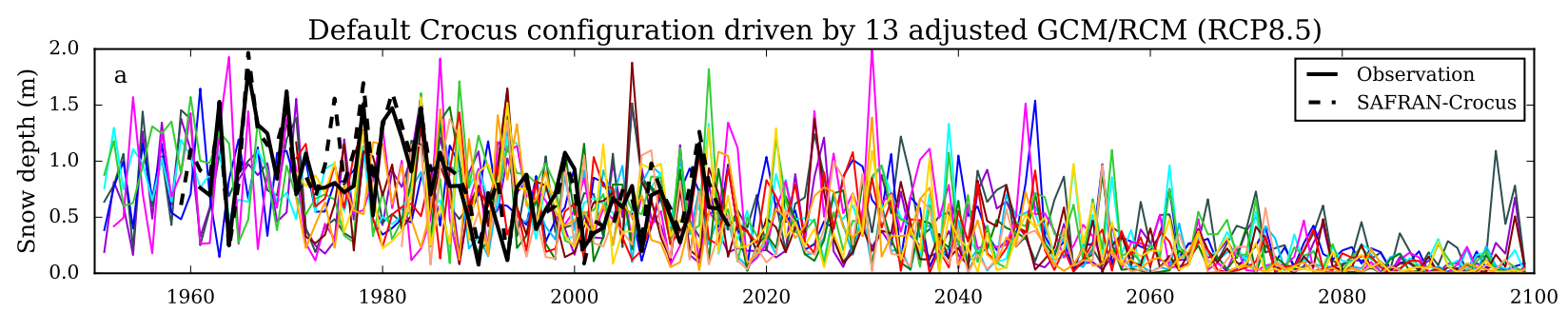

35 ESCROC members and default Crocus configuration driven by 1 adjusted GCM/RCM
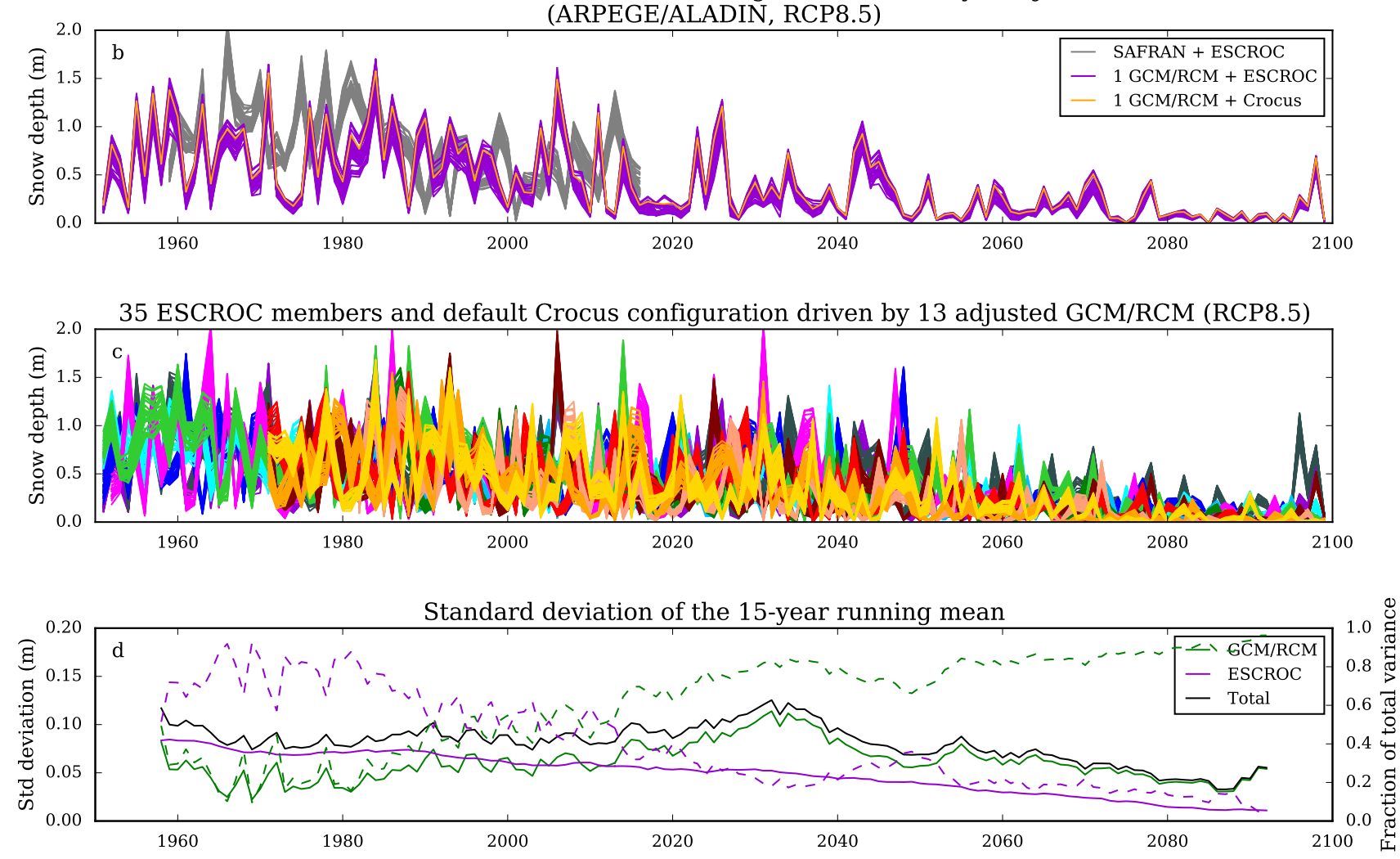

Figure 1. Observed and simulated time series of $\overline{S D}$. a) Continuous time series of annual values of mean winter snow depth data $(\overline{S D})$, either observed or generated by the default snowpack model configuration fed by meteorological data from a reanalysis or an adjusted RCM. b) $\overline{S D}$ values obtained using the ensemble of Crocus model configurations ESCROC. c) Ensemble of Crocus model configurations driven by the $13 \mathrm{RCM} / \mathrm{GCM}$ couples in the case of the RCP8.5, each RCM/GCM couple being displayed with a given color. d) Estimate of absolute and relative contribution of uncertainty components arising from RCM/GCM inter-model variability and multiphysics snowpack model uncertainty (ESCROC). 

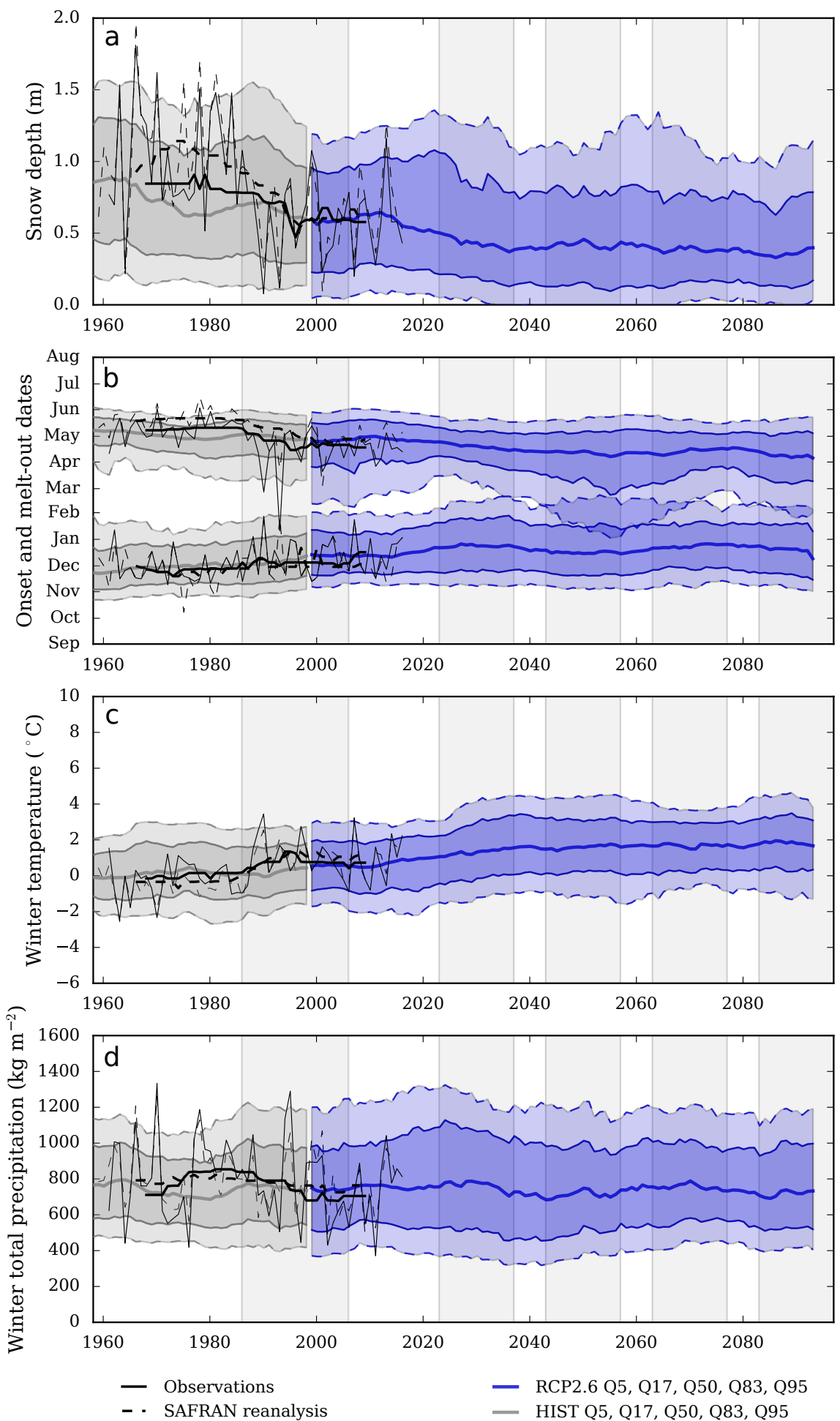

Figure 2. Quantile values (5\%, 17\%, 50\%, 83\% and 95\%) over 15-year windows of all RCM/GCM combinations among the RCP2.6 scenario, with annual values of observations (1960-2014) and SAFRAN-Crocus runs (1959-2016) and their respective 15-year running medians (bold full and dotted lines respectively) at CDP, for: a) $\overline{S D}$, b) $S O D$ and $S M O D$, c) $\bar{T}$, and d) $\bar{P}$. 

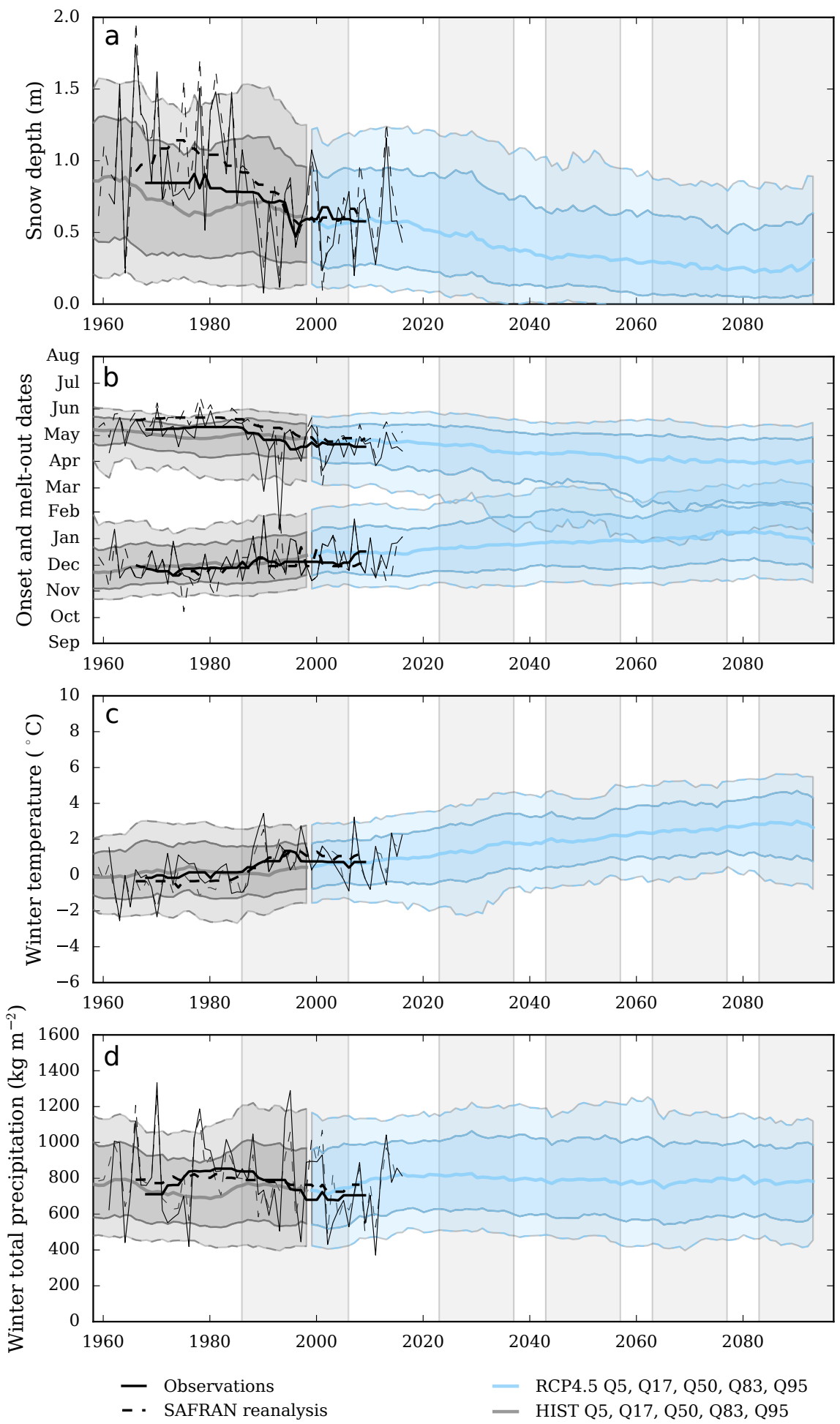

Figure 3. Quantile values (5\%, 17\%, 50\%, 83\% and 95\%) over 15-year windows of all RCM/GCM combinations among the RCP4.5 scenario, with annual values of observations (1960-2014) and SAFRAN-Crocus runs (1959-2016) and their respective 15-year running medians (bold full and dotted lines respectively) at CDP, for: a) $\overline{S D}$, b) $S O D$ and $S \mathbf{4} M O D$, c) $\bar{T}$, and d) $\bar{P}$. 

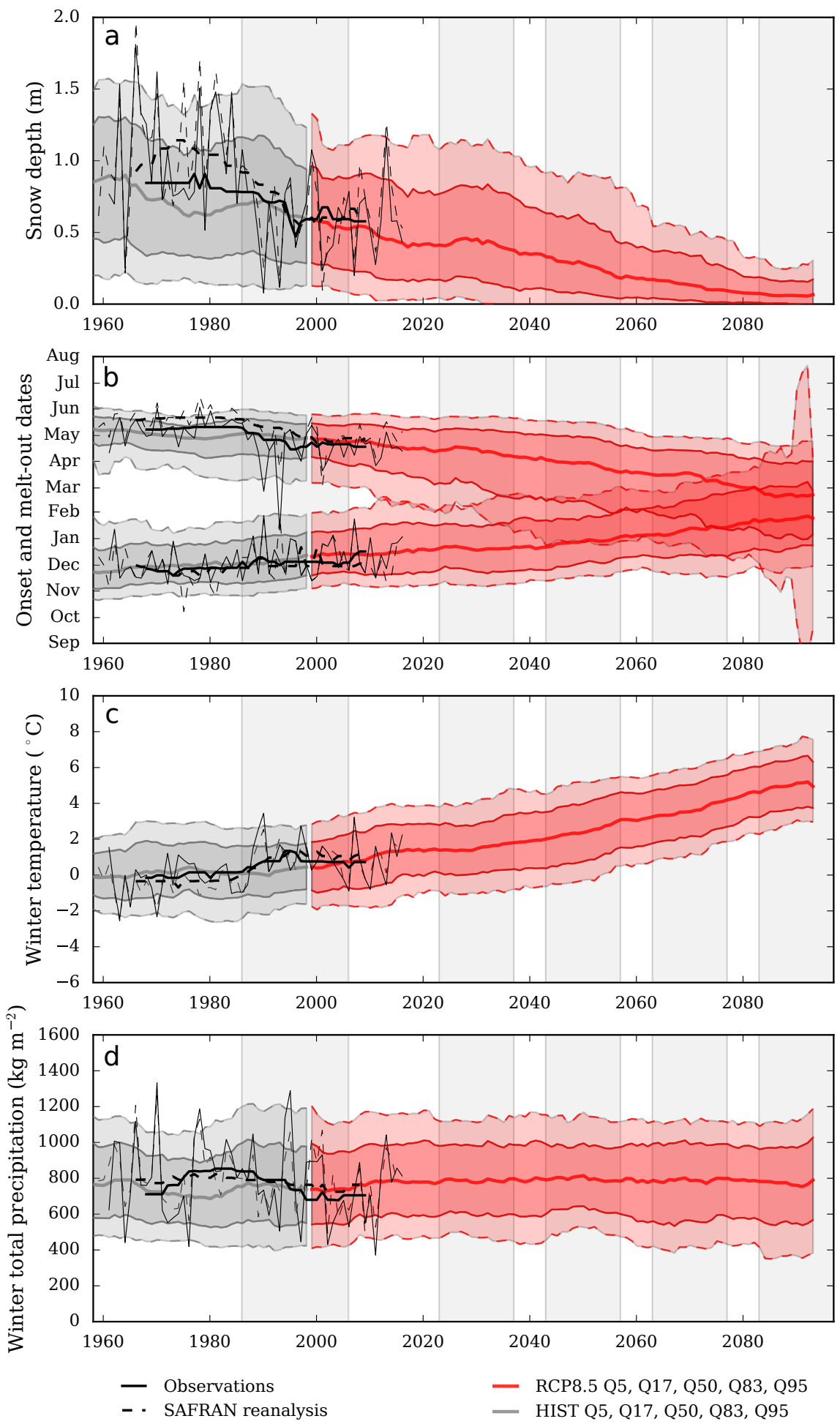

Figure 4. Quantile values (5\%, 17\%, 50\%, 83\% and 95\%) over 15-year windows of all RCM/GCM combinations among the RCP8.5 scenario, with annual values of observations (1960-2014) and SAFRAN-Crocus runs (1959-2016) and their respective 15-year running medians (bold full and dotted lines respectively) at CDP, for: a) $\overline{S D}$, b) $S O D$ and $S M O D$, c) $\bar{T}$, and d) $\bar{P}$. 

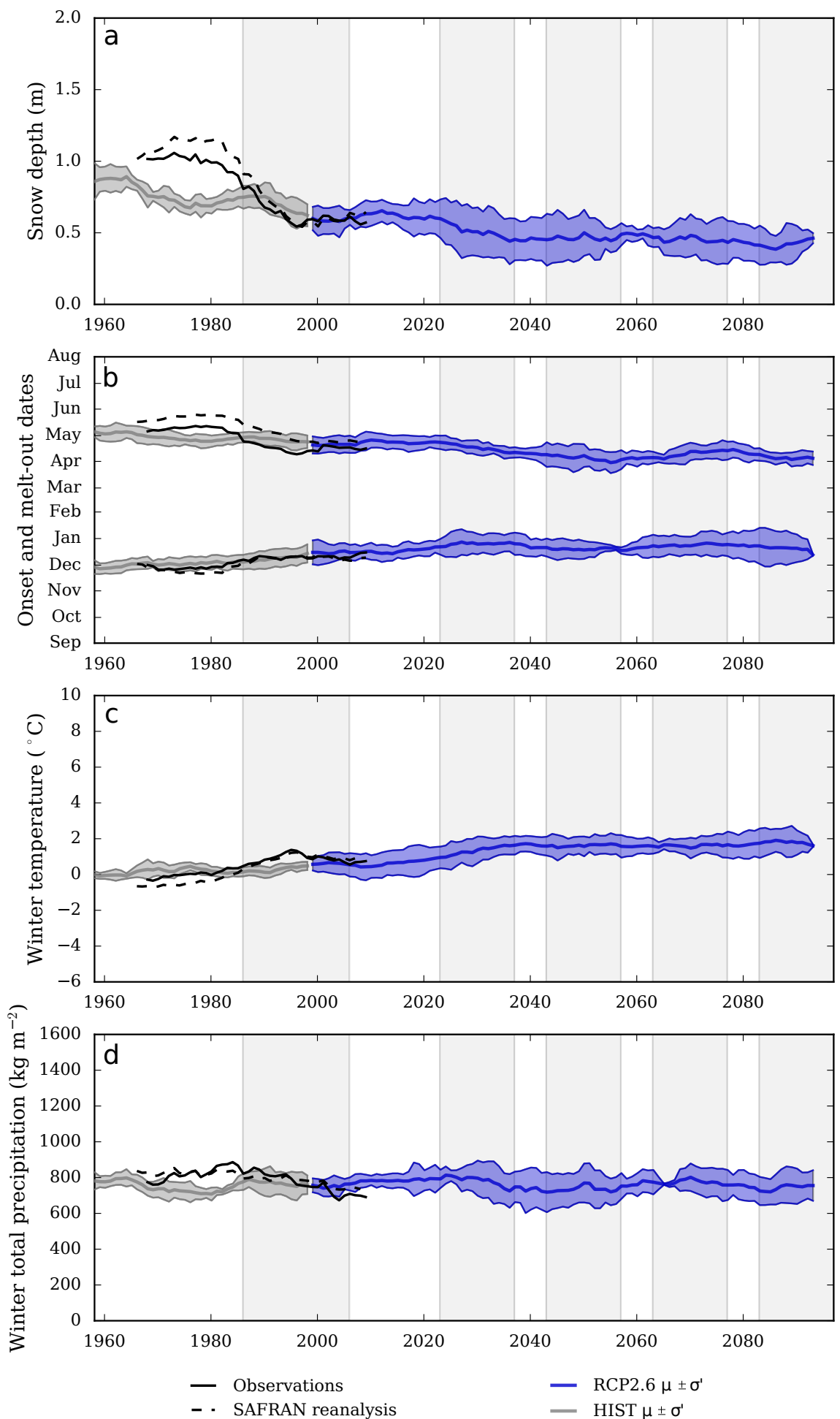

Figure 5. Mean $(\mu) \pm \sigma^{\prime}$ of all RCM/GCM combination 15-year running means among the RCP2.6 scenario, with 15-year running means of annual values of observations (1960-2014) and outputs of SAFRAN-Crocus runs (1959-2016) at CDP, for: a) $\overline{S D}$, b) $S O D$ and $S M O D$, c) $\bar{T}$, and d) $\bar{P}$. 

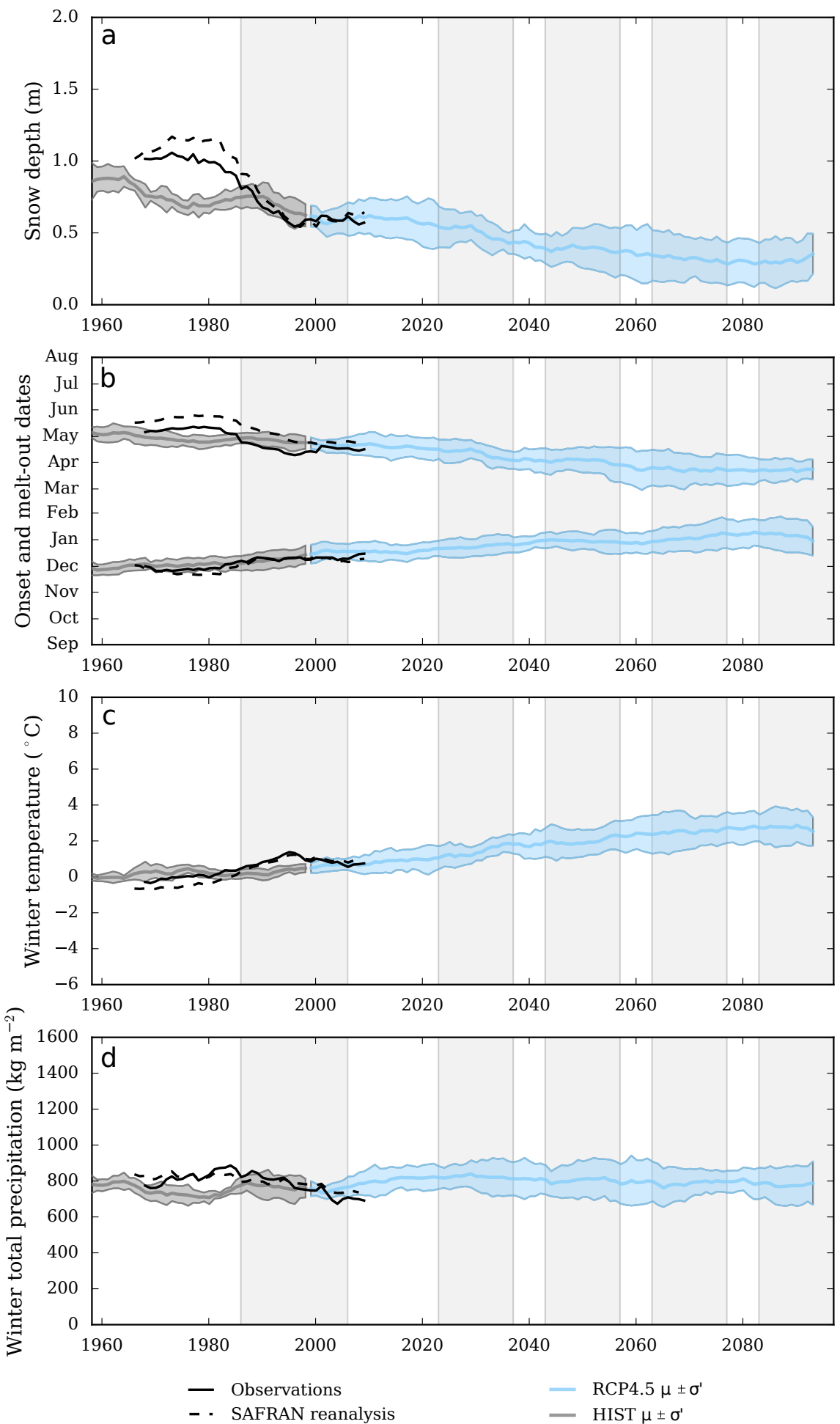

Figure 6. Mean $(\mu) \pm \sigma^{\prime}$ of all RCM/GCM combination 15-year running means among the RCP4.5 scenario, with 15-year running means of annual values of observations (1960-2014) and outputs of SAFRAN-Crocus runs (1959-2016) at CDP, for: a) $\overline{S D}$, b) $S O D$ and $S M O D$, c) $\bar{T}$, and d) $\bar{P}$. 

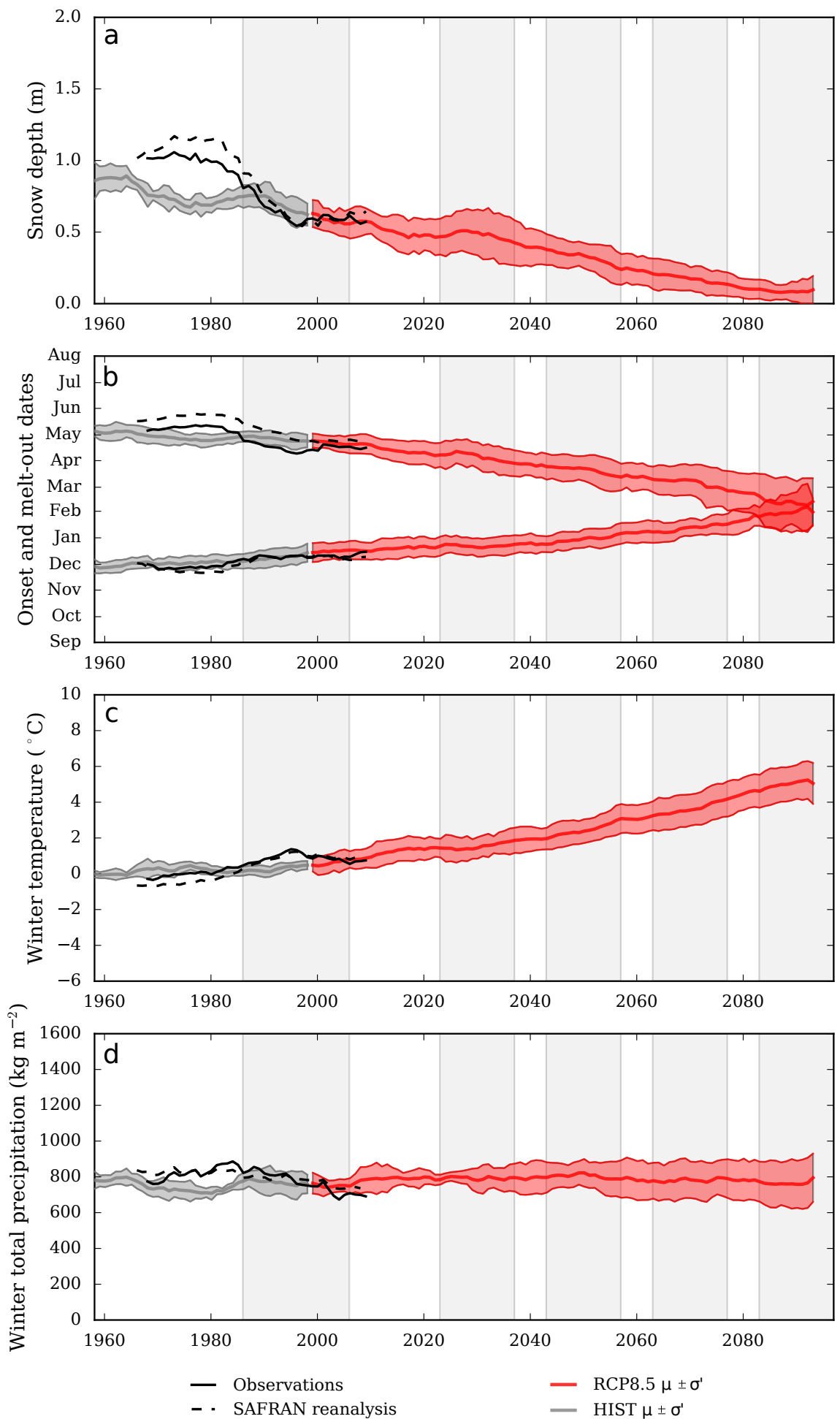

Figure 7. Mean $(\mu) \pm \sigma^{\prime}$ of all RCM/GCM combination 15-year running means among the RCP8.5 scenario, with 15 -year running means of annual values of observations (1960-2014) and outputs of SAFRAN-Crocus runs (1959-2016) at CDP, for: a) $\overline{S D}$, b) $S O D$ and $S M O D$, c) $\bar{T}$, and d) $\bar{P}$. 